\title{
Celecoxib as an Adjuvant to Fluvoxamine in Moderate to Severe Obsessive-compulsive Disorder: A Double- blind, Placebo-controlled, Randomized Trial
}

Authors

Affiliations
M. Shalbafan ${ }^{*}$, P. Mohammadinejad ${ }^{2}{ }^{*}$, S.-V. Shariat ${ }^{3}$, K. Alavi $^{3}$, A. Zeinoddini ${ }^{2}$, M. Salehi ${ }^{4}$, N. Askari ${ }^{2}$, S. Akhondzadeh ${ }^{2}$

Affiliation addresses are listed at the end of the article
Key words

anti-inflammatory agents

- celecoxib

cyclooxygenase-2 inhibitors

- obsessive-compulsive disorder

\section{Abstract}

$\nabla$

Introduction: A growing body of evidence implicates inflammatory cascades in the pathophysiology of obsessive-compulsive disorder (OCD), making this pathway a target for development of novel treatments.

Methods: 50 outpatients with moderate to severe OCD participated in the trial, and underwent 10 weeks of treatment with either celecoxib (200 mg twice daily) or placebo as an adjuvant to fluvoxamine. Participants were investigated using Yale-Brown Obsessive Compulsive Scale (Y-BOCS). The main outcome measure was to assess the efficacy of celecoxib in improving the OCD symptoms.

\section{Introduction}

$\nabla$

Obsessive-compulsive disorder (OCD) is a relatively common psychological disorder with a reported lifetime prevalence of $1-3 \%$ in the general population [1]. Currently, selective serotonin reuptake inhibitors (SRIs) and/or cognitivebehavioral therapy, particularly exposure and response prevention (ERP), are first-line medications of choice in patients with mild to moderate OCD [2,3]. Unfortunately, SRIs usually reduce the severity of OCD symptoms by as little as $20-30 \%$ [4] and only 40-60\% of OCD patients achieve satisfactory results [5-7]. Hence, an increasing number of studies have focused on the development of augmentative agents in the management of OCD [1]. There is a growing body of evidence indicating the probable role of inflammatory processes and immune dysregulation in the etiopathogenesis of OCD. OCD has been reported to be associated with autoimmune disorders triggered by inflammatory processes such as streptococcal infections [8].

\footnotetext{
${ }^{*}$ The first two authors contributed equally to this work
}

Results: General linear model repeated measures demonstrated significant effect for timex treatment interaction on the Y-BOCS total scores [F $(1.38,66.34)=6.91, p=0.005]$. Kaplan-Meier estimation with log-rank test demonstrated significantly more rapid response in the celecoxib group than the placebo group $(\mathrm{p}<0.001)$. There was no significant difference in adverse event frequencies between the groups.

Discussion: The results of the current study suggest that celecoxib could be a tolerable and effective adjunctive treatment for more rapid and more satisfying improvements in OCD symptoms.
Obsessive-compulsive behavior is also the main characteristic feature of pediatric autoimmune neuropsychiatric disorders associated with streptococcal infection (PANDAS) syndrome. Etiopathogenesis and symptom exacerbation of PANDAS have been attributed to an inappropriate immune response to streptococcal infection resulting in its central nervous system manifestations, which include OCD and tics [9]. Interestingly, while immunomodulatory therapeutic modalities including plasma exchange and intravenous immunoglobulin therapy have shown somewhat promising results in improving neuropsychiatric symptoms of PANDAS [10], there is no conclusive evidence for the probable beneficial role of antibiotics in reducing PANDAS symptoms [11] that would further indicate the role of autoimmunity in the etiopathogenesis of PANDAS. The role of autoimmune responses in the etiopathogenesis of OCD may also be supported by a decrease in the number of regulatory T cells as well as mild dysregulation of other inflammatory cell types in blood samples of OCD patients compared to age- and sex-matched healthy controls [12]. 
Along with OCD, the findings of numerous studies also suggested the role of proinflammatory cytokines in the etiopathogenesis of several neuropsychiatric disorders including schizophrenia, bipolar disorder and major depressive disorder [13]. Furthermore, investigation of the therapeutic opportunities provided by this thesis has resulted in several studies on the treatment of neuropsychiatric disorders with promising results $[14,15]$.

Celecoxib is a non-steroidal anti-inflammatory drug (NSAID) and selective inhibitor of cyclooxygenase-2 (COX-2), a known promoter of both inflammation and pain. COX-2 inhibitors have been proven to be beneficial in prevention of glutamate-mediated neuronal death and suppression of proinflammatory cytokines $[16,17]$. Celecoxib also has the advantage of reduced gastrointestinal complications compared to other NSAIDs.

Based on the currently available data, we hypothesized that the anti-inflammatory effects of celecoxib may be beneficial in the medication management of OCD. The present 10 -week, doubleblind, placebo-controlled trial aimed to evaluate the safety and efficacy of adjunctive therapy with celecoxib in improving the clinical symptoms of moderate to severe OCD.

\section{Patients and Methods}

$\nabla$

\section{Trial design and setting}

A single-center, 10-week, randomized, double-blind, placebocontrolled, parallel-group trial was conducted in the outpatient clinics of Roozbeh Psychiatric Hospital (Tehran University of Medical Sciences, Tehran, Iran) from January 2014 to November 2014. Approval was obtained from the Institutional Review Board (IRB) of Tehran University of Medical Science (Grant No: 23218). The trial was performed according to the Declaration of Helsinki and its subsequent revisions. All patients signed an informed consent prior to study entry. The trial was registered at the Iranian registry of clinical trials (www.irct.ir; registration number: IRCT201312181556N56).

\section{Participants}

Men and women, aged between 18-60 years, with a diagnosis of OCD according to the Diagnostic and Statistical Manual of Mental Disorders, Fourth Edition, Text Revision (DSM-IV-TR) and a Yale-Brown Obsessive Compulsive Scale (Y-BOCS) score of $\geq 21$ to have moderate to severe $O C D$, were eligible to take part in the study. Exclusion criteria were as follows: any other mental disorder on DSM-IV axis I, alcohol or substance (with the exception of nicotine) dependence, intelligence quotient $<70$, any serious medical illness including cardiac, hepatic and renal disease, ingestion of any psychotropic medications during the last 6 weeks, pregnancy, breast feeding and rises in liver transaminase to 3 times the upper limit of normal or higher.

\section{Interventions}

Eligible participants were randomly assigned to receive either $200 \mathrm{mg}$ celecoxib (celebrex, Pfizer, $200 \mathrm{mg}$ capsule) twice daily or placebo in the same manner for 10 weeks. All patients, regardless of their treatment group, also received fluvoxamine $100 \mathrm{mg} /$ day for the first 4 weeks of the study followed by $200 \mathrm{mg} /$ day for the rest of the trial course.

\section{Outcomes}

All patients were assessed using Y-BOCS at baseline and weeks 4 and 10. A psychiatrist with good experience in implementing the Y-BOCS who had been involved in several clinical trials was in charge of rating the patients. The main outcome measure of this trial was evaluation of celecoxib efficacy in improvement of Y-BOCS total score compared to placebo using the general linear repeated measure model. Partial response and complete response were defined as $25 \%$ and $35 \%$ reduction in the Y-BOCS score, respectively. A Y-BOCS score $<16$ was considered as remission. Adverse events were systematically evaluated at each time point.

\section{Sample size}

Assuming a difference of 4 on the Y-BOCS total score between the celecoxib group and the placebo group with a standard deviation (SD) of 4.5 , a power of $80 \%$, and a two-sided significance level of $5 \%$, a sample size of 42 was needed. Considering a $20 \%$ attrition rate, a final sample size of 54 was planned.

\section{Randomization, allocation, concealment and blinding}

Generation of randomization codes was performed by a computerized random number generator (blocks of 4, allocation ratio 1:1). Concealment of allocation was performed using sequentially numbered and sealed packages. Separate people were responsible for random allocation and rating of the patients. The patients, research investigators, rater and the statistician were all blinded to the allocated treatment. Celecoxib and placebo capsules were completely identical in their shape, color, size, texture, and odor. The placebo rate anticipation was also assessed by the participants, and the rater at the study end.

\section{Statistical analysis}

All analyses were carried out according to the intention-to-treat principles with at least one post-baseline measurement. Continuous variables were reported as mean \pm standard deviation (SD) and categorical variables were reported as frequency (percentage). Baseline continuous variables were compared using an independent t-test. A general linear model repeated measure was used to evaluate time $\times$ treatment interaction considering the treatment group (celecoxib vs. placebo) as the between-subject factor and the study measurements as the within-subject factor (time). Greenhouse-Geisser adjustment in degrees of freedom was made if Mauchly's test of sphericity was significant. An independent t-test and Cohen's d effect size were used to compare score change from baseline to each time interval between the 2 study groups. Categorical variables were compared using chi-square test or Fisher's exact test as appropriate. The time needed to respond to treatment was compared between the celecoxib and the placebo groups using KaplanMeier estimation with log-rank test. Statistical Package of Social Science Software (SPSS version 20, IBM Company, Armonk NY, USA) was used for statistical analysis and the graphs were drawn using SigmaPlot (version 12).

\section{Results}

$\nabla$

\section{Participants and baseline characteristics}

A total of 104 participants screened for eligibility criteria, among them 54 patients, were randomly assigned to receive either celecoxib plus fluvoxamine $(\mathrm{n}=27)$ or placebo plus fluvoxamine $(n=25) .50$ patients (25 in each group) had at least one postbaseline measurement and a similar number of study participants completed the trial ( $\bullet$ Fig. 1). Baseline characteristics of 
the patients were not significantly different between the groups (॰ Table 1).

\section{Outcome}

\section{Y-BOCS total score}

General linear model repeated measures demonstrated significant effect for time $\times$ treatment interaction on Y-BOCS total score during the trial course $[F(1.38,66.34)=6.91, p=0.005](\otimes$ Fig. $2 a)$. At the study conclusion, remission achieved in $15(60 \%)$ patients in the celecoxib group was compared with $8(32 \%)$ patients in the placebo group $(\mathrm{p}<0.047)$. Significantly, higher partial and complete response rates were also observed in the celecoxib group than the placebo group at the end of the trial ( $\bullet$ Table 2 ). An independent t-test demonstrated significantly greater reduction in Y-BOCS total score in the celecoxib group than the placebo group at weeks 4 and 10 ( $\odot$ Table 3 ). Kaplan-Meier estimation showed that a shorter time was needed in the celecoxib group than the placebo group for partial response, $\mathrm{p}<0.001$.

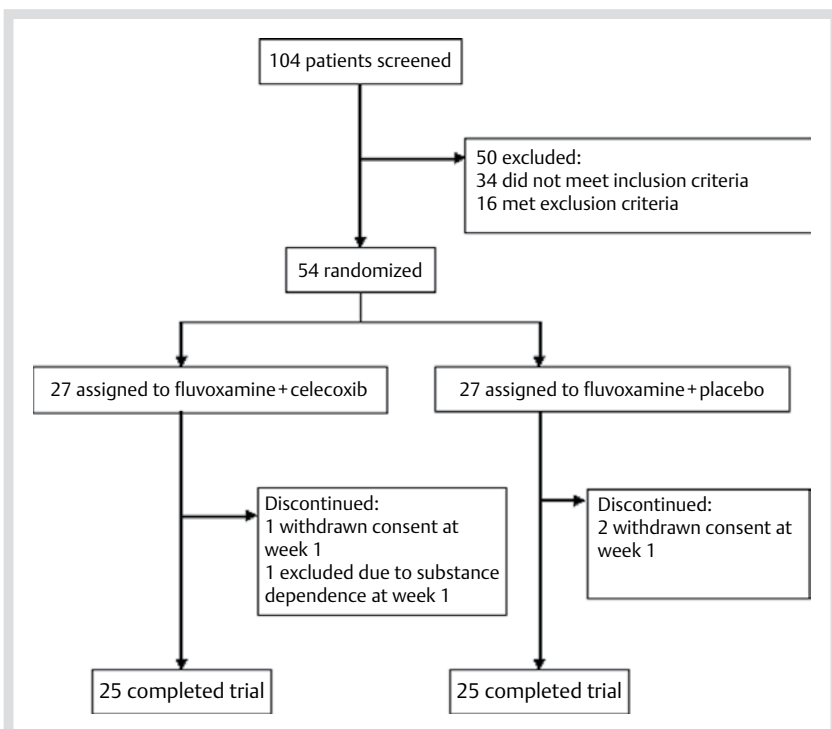

Fig. 1 Flow diagram of the trial.

\section{Y-BOCS obsession subscale score}

General linear model repeated measures demonstrated significant effect for time $\times$ treatment interaction on Y-BOCS total score during the trial course $[F(1.44,69.33)=7.17, p=0.004]$ ( $\odot$ Fig. $2 b$ ). An independent t-test demonstrated significantly greater reduction in Y-BOCS obsession subscale scores in the celecoxib group than the placebo group at weeks 4 and 10 ( $\odot$ Table 3 ).

\section{Y-BOCS compulsion subscale score}

General linear model repeated measures demonstrated significant effect for time $\times$ treatment interaction on Y-BOCS total score during the trial course $[\mathrm{F}(1.35,64.90)=3.99, \mathrm{p}=0.038]$ ( $\odot$ Fig. 2c). Independent t-test demonstrated significantly greater reduction in Y-BOCS obsession subscale scores in the celecoxib group than the placebo group at weeks 10 (॰ Table 3 ).

\section{Adverse events}

Frequency of adverse events did not differ significantly between treatment groups ( $\odot$ Table 4 ). No serious adverse events or deaths occurred.

\section{Blinding}

The participants and the rater were unsure about the treatment allocation in more than $50 \%$ of the allocations.

\section{Discussion}

$\nabla$

The results of the current study showed that the administration of celecoxib, as an adjuvant agent in addition to fluvoxamine, is significantly superior to fluvoxamine monotherapy in reducing both obsessive and compulsive symptoms and achieving a more rapid response to treatment in patients with moderate to severe OCD. The use of celecoxib also appeared to be safe and well tolerated in our study population and no clinically significant adverse effect was reported. The response to treatment in the placebo arm who received monotherapy with fluvoxamine was similar to the previous reports of treatment outcome with SRIs in OCD patients [4]. Fluvoxamine monotherapy resulted in about $30 \%$ reduction in OCD symptoms (9.4 points on Y-BOCS total score). Celecoxib plus fluvoxamine resulted in about $50 \%$ reduc-

\begin{tabular}{|ll|}
\hline Variable & Celecoxib group $(\mathbf{n = 2 5})$ \\
\hline age, years, mean \pm SD & $33.28 \pm 8.64$ \\
\hline weight $(\mathrm{kg})$ & $79.3 \pm 11.2$ \\
\hline height $(\mathrm{cm})$ & $171.6 \pm 14.3$ \\
\hline sex, female, $\mathrm{n}(\%)$ & $9(36 \%)$ \\
\hline duration of the disease (years), mean \pm SD & $5.12 \pm 2.87$ \\
\hline single: married & $8(32 \%): 17(68 \%)$ \\
\hline education & \\
\hline - primary or secondary school & $8(32 \%)$ \\
\hline - diploma & $13(52 \%)$ \\
\hline - university degree & $4(16 \%)$ \\
\hline Y-BOCS total score, mean \pm SD & $30.24 \pm 3.87$ \\
\hline Y-BOCS obsession subscale, mean \pm SD & $16.20 \pm 2.14$ \\
\hline Y-BOCS compulsion subscale, mean \pm SD & $14.04 \pm 2.59$ \\
\hline baseline HDRS score, mean \pm SD & $7.24 \pm 1.12$ \\
\hline medications history & Fluoxetine: $15 ;$ \\
& Clomipramine: $10 ;$ \\
\hline
\end{tabular}

$\begin{array}{ll}\text { Placebo group }(\mathbf{n}=\mathbf{2 5}) & \text { P-value } \\ 31.16 \pm 8.90 & 0.40 \\ 78.1 \pm 11.4 & 0.70 \\ 172.8 \pm 12.2 & 0.71 \\ 10(40 \%) & 0.77 \\ 6.40 \pm 3.74 & 0.18 \\ 10(40 \%): 15(60 \%) & 0.77 \\ & 0.13 \\ 5(20 \%) & \\ 9(36 \%) & \\ 11(44 \%) & \\ 29.48 \pm 1.69 & 0.37 \\ 15.36 \pm 1.35 & 0.10 \\ 14.12 \pm 1.01 & 0.89 \\ \text { 6.98 } \pm 1.48 & 0.48 \\ \text { Fluoxetine: } 16 ; & \\ \text { Clomipramine: } 12 ; & \\ \text { Fluvoxamine: } 10 & \\ \text { Th } & \end{array}$

n, number; SD, standard deviation; Y-BOCS: Yale-Brown Obsessive Compulsive Scale; HDRS, Hamilton depression rating scale
Table 1 Baseline characteristics according to the treatment group. 


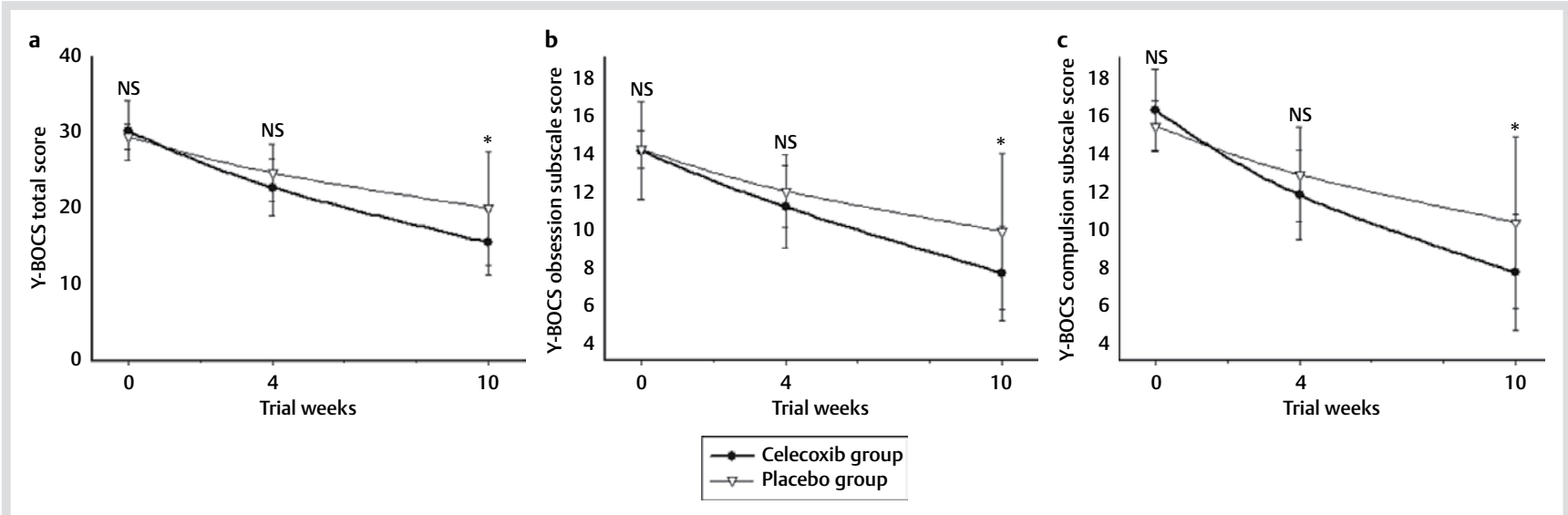

Fig. 2 Repeated-measure analysis of variance for the effect of 2 treatments on the Yale-Brown Obsessive Compulsive Scale (Y-BOCS) total a, obsession $\mathbf{b}$ and compulsion $\mathbf{c}$ scores (mean $\pm \mathrm{SD}$; ${ }^{*} \mathrm{P}<0.05$ ). NS, not significant.

\begin{tabular}{|lcllll|}
\hline Y-BOCS score & $\begin{array}{l}\text { Celecoxib } \\
\text { group } \mathbf{( n = 2 5 )}\end{array}$ & $\begin{array}{l}\text { Placebo } \\
\text { group (n=25) }\end{array}$ & $\begin{array}{l}\text { Mean difference celecoxib- } \\
\text { placebo (95\% Cl) }\end{array}$ & $\begin{array}{l}\text { Cohen' } \\
\text { d }\end{array}$ & P-value \\
\hline total (week 4) & $7.44 \pm 4.04$ & $4.76 \pm 3.91$ & $2.68(0.42-4.94)$ & 0.67 & 0.02 \\
\hline total (week 10) & $14.68 \pm 4.82$ & $9.40 \pm 7.80$ & $5.28(1.57-8.99)$ & 0.81 & 0.006 \\
\hline obsession (week 4) & $4.48 \pm 2.61$ & $2.56 \pm 2.53$ & $1.92(0.46-3.38)$ & 0.74 & 0.01 \\
\hline obsession (week 10) & $8.56 \pm 3.74$ & $5.08 \pm 4.50$ & $3.48(1.13-5.83)$ & 0.84 & 0.005 \\
\hline compulsion (week 4) & $2.96 \pm 1.93$ & $2.20 \pm 1.87$ & $0.76(-0.32-1.84)$ & 0.40 & 0.16 \\
\hline compulsion (week 10) & $6.44 \pm 2.08$ & $4.32 \pm 4.44$ & $2.12(0.13-4.11)$ & 0.61 & 0.04 \\
\hline
\end{tabular}

Table 2 Comparison of changes in the Yale-Brown Obsessive Compulsive Scale total and subscales scores from baseline between the 2 groups using independent t-test.

$\mathrm{Cl}$, confidence interval; $\mathrm{n}$ number; Y-BOCS, Yale-Brown Obsessive Compulsive Scale
Table 4 The frequency of different types of adverse events in the study population.

\begin{tabular}{|llll|}
\hline Adverse event & $\begin{array}{l}\text { Celecoxib } \\
\text { group (n=25) }\end{array}$ & $\begin{array}{l}\text { Placebo } \\
\text { group (n=25) }\end{array}$ & P-value \\
\hline increased appetite, n, \% & $8(32)$ & $3(12)$ & 0.09 \\
\hline daytime drowsiness, n, \% & $7(28)$ & $5(20)$ & 0.51 \\
\hline slowed movement, n, \% & $7(28)$ & $2(8)$ & 0.065 \\
\hline morning drowsiness, n, \% & $6(24)$ & $3(12)$ & 0.46 \\
\hline dizziness, n, \% & $5(20)$ & $4(16)$ & 1.00 \\
\hline nervousness, n, \% & $5(20)$ & $2(8)$ & 0.42 \\
\hline restlessness, n, \% & $4(16)$ & $3(12)$ & 1.00 \\
\hline dry mouth, n, \% & $4(16)$ & $5(20)$ & 1.00 \\
\hline fatigue, n, \% & $3(12)$ & $3(12)$ & 1.00 \\
\hline diarrhea, n, \% & $3(12)$ & $0(0)$ & 0.23 \\
\hline abdominal pain, n, \% & $3(12)$ & $3(9)$ & 1.00 \\
\hline Yyawning, n, \% & $3(12)$ & $5(20)$ & 0.70 \\
\hline headache, n, \% & $3(12)$ & $4(16)$ & 1.00 \\
\hline twitches, n, \% & $2(8)$ & $3(12)$ & 1.00 \\
\hline loss of appetite, n, \% & $1(4)$ & $5(20)$ & 0.19 \\
\hline
\end{tabular}

tion in OCD symptoms (14.7 points on Y-BOCS total score) by the trial conclusion. Since baseline characteristics of patients in the 2 study groups did not differ significantly, it cannot explain the differences observed in the efficacy of fluvoxamine and placebo as adjuvant agents.

The findings of the present study are consistent with the report of Sayyah et al. on augmentative treatment with celecoxib in patients with OCD. They investigated the efficacy of the same celecoxib dosage as an adjuvant therapy to fluoxetine in the management of OCD in an 8-week clinical trial [18]. They found that those in the celecoxib group showed a greater improvement of OCD symptoms compared to controls who received fluoxetine and placebo. The superior effect of celecoxib compared to placebo was observed as soon as 2 weeks after the initiation of therapy and was still significant at the study end at week 8 . In agreement with their report, we found a significantly greater improvement of OCD symptoms in the first assessment after receiving medication for 4 weeks and the results were repeated at week 10. The beneficial effects of celecoxib in rapid reduction of obsessive and compulsive symptoms may be explained mainly by the role of COX-2 inhibitors in the suppression of inflamma- 
tory processes in the brain. COX-2 plays an important role in the initiation of inflammatory processes by catalyzing the conversion of arachidonic acid to prostaglandin H2 [19]. Prostaglandin $\mathrm{H} 2$ itself is a precursor for other biologically active molecules involved in further regulation of inflammatory cascades including prostaglandin $\mathrm{D} 2$, prostaglandin E2, prostacyclin and thromboxane A2 [20]. Most recently, Konuk et al. evaluated the plasma levels of 2 proinflammatory cytokines, interleukin-6 (IL-6) and tumor necrosis factor(TNF)- $\alpha$, among OCD patients with no major neuropsychological disorder who had not have received any psychotropic agents or NSAIDs for at least 3 months prior to blood sampling [21]. They reported higher plasma levels of both IL- 6 and TNF- $\alpha$ in OCD patients compared to healthy controls. Moreover, they found a negative correlation between TNF- $\alpha$ level and the onset of disease as well as a weak correlation between IL- 6 levels and duration of illness. The authors suggested that new therapeutic modalities can be developed based on the speculated role of inflammation in the etiopathogenesis of OCD. Nevertheless, a recent meta-analysis showed that overall plasma levels of IL-1 $\beta$ are reduced in OCD patients relative to controls, while there is no overall difference in TNF- $\alpha$ and IL-6 plasma levels [22]. Interestingly, celecoxib is known to significantly reduce elevated levels of both IL- 6 and TNF- $\alpha$, which further supports the importance of its anti-inflammatory characteristics in improving clinical symptoms of OCD patients $[14,15]$.

In the present study, celecoxib was administrated with a dosage of $200 \mathrm{mg}$ twice daily with no serious adverse events. We have already reported the safety and tolerability of this practice in several previous studies $[14,15]$. We did not witness any serious cardiovascular adverse effects here. Indeed, the lack of such findings can be attributed to the low doses of celecoxib administered, short duration of therapy and follow-up, and relatively small number of patients investigated. Limitations of the present study include: small sample size, short follow-up period and no assessment of proinflammatory cytokines.

In conclusion, the results of the current study suggest that the administration of celecoxib as an adjuvant agent with SRIs might help in achieving rapid and more satisfying improvements in OCD symptoms. Further randomized controlled trials with larger sample sizes, longer study duration, and proinflammatory cytokine assessment are warranted in order to confirm the beneficial effects of celecoxib in long-term management of OCD.

\section{Source of Funding}

$\nabla$

This study was supported by a grant from the Tehran University of Medical Sciences to Professor Shahin Akhondzadeh (Grant No. 23218).

\section{Acknowledgement}

$\nabla$

This study was Dr Mohammadreza Shalbafan's postgraduate thesis toward qualification for the Iranian Board of Psychiatry under the supervision of Professor Shahin Akhondzadeh.

\section{Conflict of Interest}

$\nabla$

The authors declare no conflict of interest.
Affiliations

School of Medicine, Iran University of Medical Sciences, Tehran, Iran

2 Psychiatric Research Center, Roozbeh Hospital, Tehran University of Medical Sciences, Tehran, Iran

${ }^{3}$ Mental Health Research Center, Tehran Institute of Psychiatry, School of Behavioral Sciences and Mental Health, Iran University of Medical Sciences, Tehran, Iran

${ }^{4}$ Tehran Institute of Psychiatry, School of Behavioral Sciences and Mental Health, Iran University of Medical Sciences, Tehran, Iran

\section{References}

1 Heidari M, Zarei M, Hosseini SM et al. Ondansetron or placebo in the augmentation of fluvoxamine response over 8 weeks in obsessivecompulsive disorder. Int Clin Psychopharmacol 2014; 29: 344-350

2 Seibell PJ, Hollander E. Management of obsessive-compulsive disorder. F1000Prime Rep 2014; 6: 68

3 Foa EB, Liebowitz MR, Kozak MJ et al. Randomized, placebo-controlled trial of exposure and ritual prevention, clomipramine, and their combination in the treatment of obsessive-compulsive disorder. Am J Psychiatry 2005; 162: 151-161

4 Pigott TA, Seay SM. A review of the efficacy of selective serotonin reuptake inhibitors in obsessive-compulsive disorder. J Clin Psychiatry 1999; 60: 101-106

5 Erzegovesi S, Cavallini MC, Cavedini P et al. Clinical predictors of drug response in obsessive-compulsive disorder. J Clin Psychopharmacol 2001; 21: 488-492

6 Askari N, Moin M, Sanati $M$ et al. Granisetron adjunct to fluvoxamine for moderate to severe obsessive-compulsive disorder: a randomized, double-blind, placebo-controlled trial. CNS Drugs 2012; 26: 883-892

7 Ghaleiha A, Entezari N, Modabbernia A et al. Memantine add-on in moderate to severe obsessive-compulsive disorder: randomized double-blind placebo-controlled study. J Psychiatr Res 2013; 47: 175-180

8 Hounie AG, Cappi C, Cordeiro Q et al. TNF-alpha polymorphisms are associated with obsessive-compulsive disorder. Neurosci Lett 2008; 442: 86-90

9 Snider LA, Swedo SE. Post-streptococcal autoimmune disorders of the central nervous system. Curr Opin Neurol 2003; 16: 359-365

10 Perlmutter SJ, Leitman SF, Garvey MA et al. Therapeutic plasma exchange and intravenous immunoglobulin for obsessive-compulsive disorder and tic disorders in childhood. Lancet 1999; 354: 1153-1158

11 Garvey MA, Perlmutter SJ, Allen AJ et al. A pilot study of penicillin prophylaxis for neuropsychiatric exacerbations triggered by streptococcal infections. Biol Psychiatry 1999; 45: 1564-1571

12 Kawikova I, Leckman JF, Kronig $H$ et al. Decreased numbers of regulatory $\mathrm{T}$ cells suggest impaired immune tolerance in children with Tourette syndrome: a preliminary study. Biol Psychiatry 2007; 61: 273-278

13 Mitchell RH, Goldstein BI. Inflammation in children and adolescents with neuropsychiatric disorders: a systematic review. J Am Acad Child Adolesc Psychiatry 2014; 53: 274-296

14 Akhondzadeh $S$, Jafari $S$, Raisi $F$ et al. Clinical trial of adjunctive celecoxib treatment in patients with major depression: a double blind and placebo controlled trial. Depress Anxiety 2009; 26: 607-611

15 Abbasi SH, Hosseini F, Modabbernia A et al. Effect of celecoxib add-on treatment on symptoms and serum IL- 6 concentrations in patients with major depressive disorder: randomized double-blind placebocontrolled study. J Affect Disord 2012; 141: 308-314

16 Kimura $T$, Iwase $M$, Kondo $G$ et al. Suppressive effect of selective cyclooxygenase-2 inhibitor on cytokine release in human neutrophils. Int Immunopharmacol 2003; 3: 1519-1528

17 Strauss KI, Marini AM. Cyclooxygenase-2 inhibition protects cultured cerebellar granule neurons from glutamate-mediated cell death. J Neurotrauma 2002; 19: 627-638

18 Sayyah $M$, Boostani $H$, Pakseresht $S$ et al. A preliminary randomized double-blind clinical trial on the efficacy of celecoxib as an adjunct in the treatment of obsessive-compulsive disorder. Psychiatry Res 2011; 189: 403-406

19 Vardeh D, Wang D, Costigan $M$ et al. COX2 in CNS neural cells mediates mechanical inflammatory pain hypersensitivity in mice. J Clin Invest 2009; 119: 287-294

20 Kunz T, Marklund N, Hillered L et al. Cyclooxygenase-2, prostaglandin synthases, and prostaglandin $\mathrm{H} 2$ metabolism in traumatic brain injury in the rat. J Neurotrauma 2002; 19: 1051-1064

21 Konuk N, Tekin IO, Ozturk $U$ et al. Plasma levels of tumor necrosis factor-alpha and interleukin-6 in obsessive compulsive disorder. Mediators Inflamm 2007200765704

22 Gray SM, Bloch $M H$. Systematic review of proinflammatory cytokines in obsessive compulsive disorder. Curr Psychiatry Rep 2012; 14: 220-228 